\title{
The therapeutic value of SC66 in human renal cell carcinoma cells
}

\author{
Ming Xu', Yin Wang ${ }^{2}$, Li-Na Zhou ${ }^{3}$, Li-jun Xu', Zhi-chang Jin", Dong-rong Yang ${ }^{1}$, Min-bin Chen ${ }^{3}$ and Jin Zhu ${ }^{1}$
}

\begin{abstract}
The PI3K-AKT-mTOR cascade is required for renal cell carcinoma (RCC) progression. SC66 is novel AKT inhibitor. We found that SC66 inhibited viability, proliferation, migration and invasion of RCC cell lines (786-O and A498) and patientderived primary RCC cells. Although SC66blocked AKT-mTORC1/2 activation in RCC cells, it remained cytotoxic in AKTinhibited/-silenced RCC cells. In RCC cells, SC66 cytotoxicity appears to occur via reactive oxygen species (ROS) production, sphingosine kinase 1inhibition, ceramide accumulation and JNK activation, independent of AKT inhibition. The ROS scavenger N-acetylcysteine, the JNK inhibitor (JNKi) and the anti-ceramide sphingolipid sphingosine-1phosphate all attenuated SC66-induced cytotoxicity in 786-O cells. In vivo, oral administration of SC66 potently inhibited subcutaneous 786-O xenograft growth in SCID mice. AKT-mTOR inhibition, SphK1 inhibition, ceramide accumulation and JNK activation were detected in SC66-treated 786-O xenograft tumors, indicating that SC66 inhibits RCC cell progression through AKT-dependent and AKT-independent mechanisms.
\end{abstract}

\section{Introduction}

Renal cell carcinoma (RCC) is the most common type of renal malignancy ${ }^{1}$. Nephroureterectomy of early-stage RCC is the only possible curable treatment option ${ }^{1}$. However, RCC is more often diagnosed at an advanced stage, with $25 \%$ of patients developing local invasion and systematic metastasis resulting in a poor prognosis ${ }^{1}$. The PI3K-AKT-mTOR signaling pathway is frequently upregulated in RCC due to mechanisms that include PTEN mutation/depletion, PI3KCA mutation, and sustained activation of receptor tyrosine kinases (RTKs) $)^{2-5}$. Constitutive activation of this cascade is necessary for RCC cell proliferation, survival, migration, and metastasis, and also angiogenesis and resistance to anti-tumor treatments $2,3,6,7$. Molecularly-targeted agents are currently being utilized for the treatment of certain RCC patients,

Correspondence: Dong-rong Yang (doc_ydr@163.com) or Min-

bin Chen (cmb1981@163.com) or Jin Zhu (urologistzhujin@163.com)

${ }^{1}$ Department of Urology, The Second Affiliated Hospital of Soochow University,

Suzhou, China

${ }^{2}$ Institute of Neuroscience, Soochow University, Suzhou, China

Full list of author information is available at the end of the article

These authors contributed equally: Ming Xu, Yin Wang, Li-Na Zhou, Li-jun Xu

Edited by A. Stephanou including the mTORC1 inhibitors Temsirolimus and everolimus, which are approved by the FDA for the treatment of advanced $\mathrm{RCC}^{2,3,6,7}$.

Our group has previously shown that targeted inhibition of the PI3K-AKT-mTOR pathway is a valid treatment strategy in the management of $\mathrm{RCC}^{8-10}$. SF2523, a PI3K$\mathrm{AKT}$ and bromodomain-containing protein 4 (BRD4) dual inhibitor, was found to potently inhibit RCC cell growth in vitro and in vivo ${ }^{8}$. Similarly, the AKTmTORC1/2 inhibitor WYE-687 inhibited cell growth of human RCC cells ${ }^{9}$. In addition, we identified that microRNA-302c inhibited RCC cell proliferation by targeting Grb2-associated binding 2 (Gab2)-AKT signaling ${ }^{10}$.

Jo et al., developed $\mathrm{SC} 66^{11}$, a novel allosteric AKT inhibitor that exerted a dual-inhibitory mechanism by inducing AKT ubiquitination and interfering with AKT pleckstrin homology $(\mathrm{PH})$ domain binding to $\mathrm{PIP} 3^{11}$. The study by Cusimano et al., demonstrated that AKT inhibition by SC66 induced significant cytotoxic effects in hepatocellular carcinoma (HCC) cells ${ }^{12}$. In this study, we demonstrate that in addition to AKT-dependent RCC cell

(c) (i) Open Access This article is licensed under a Creative Commons Attribution 4.0 International License, which permits use, sharing, adaptation, distribution and reproduction cc. in any medium or format, as long as you give appropriate credit to the original author(s) and the source, provide a link to the Creative Commons license, and indicate if changes were made. The images or other third party material in this article are included in the article's Creative Commons license, unless indicated otherwise in a credit line to the material. If material is not included in the article's Creative Commons license and your intended use is not permitted by statutory regulation or exceeds the permitted use, you will need to obtain permission directly from the copyright holder. To view a copy of this license, visit http://creativecommons.org/licenses/by/4.0/. 
inhibition, SC66 inhibits RCC cell progression via AKTindependent mechanisms.

\section{Materials and methods \\ Reagents and chemicals}

SC66, MK-2206, and LY294002 were purchased from MCE Chemicals (Shanghai, China). N-acetylcysteine (NAC), PD98059, U0126, sphingosine-1-phosphate (S1P) and the JNK inhibitor (JNKi) were provided by Sigma-Aldrich (St. Louis, Mo). The broad caspase inhibitor $\mathrm{z}$-VAD-cho and the caspase-3 inhibitor z-DEVD-cho were obtained from Enzo Life Sciences (Shanghai, China). Antibodies for phosphorylated ("p")-AKT (Ser-473) (\#9271), AKT1/2 (\#9272), p-S6K1 (\#9234), S6K1 (9202), p-Erk1/2 (\#9101), Erk1/2 (\#9102), pJNK (\#9255), JNK1/2 (\#9252), SphK1 (\#12071), cleavedcaspase-3 (\#9664), cleaved-caspase-9 (\#20750), cleaved-poly (ADP-ribose) polymerase (PARP) (\#5625), Bcl-2 (\#15707), and $\beta$-tubulin (\#15115) were purchased from Cell Signaling Tech (Beverly, MA).

\section{Cell culture}

The established RCC cells (786-O and A489 lines) and immortalized HK-2 tubule epithelial cells ${ }^{13,14}$ were cultured using the previous protocol ${ }^{10,15}$. Cells were routinely subjected to mycoplasma and microbial contamination examination. STR profiling, population doubling time, and morphology were routinely checked every 3-4 months to confirm the genotype. The primary human RCC cells, derived from three primary RCC patients ("RCC1/2/3"), as well as the primary human renal epithelial cells ("Ren-Epi") were cultured in the described medium $^{8,9}$. The written-informed consent was obtained from each enrolled patient. All investigations were conducted according to the principles expressed in the Declaration of Helsinki. Experiments and protocols were approved by the Ethics Review Board of Soochow University (Suzhou, China).

\section{Methylthiazol tetrazolium (MTT) assay}

Cells were seeded onto the 96-well tissue culture plates $\left(3 \times 10^{3}\right.$ cells per well). Following treatment, cell viability was assessed by the MTT assay. MTT OD was recorded at $490 \mathrm{~nm}$.

\section{Soft agar colony formation assay}

Cells were seeded onto the 10-cm tissue culture dishes $\left(1 \times 10^{4}\right.$ cells per dish), treated with SC66 every two days for five rounds. Afterwards, the number of viable 786-O colonies were counted.

\section{BrdU assay}

Cells were seeded onto the six-well tissue culture plates $\left(1 \times 10^{5}\right.$ cells per well). Following treatment, cells were incubated with BrdU (10 $\mu$ M, Cell Signaling Tech) for $8 \mathrm{~h}$ and then fixed. BrdU incorporation was determined in the ELISA format. BrdU OD at $405 \mathrm{~nm}$ was recorded.

\section{EdU assay of cell proliferation}

Cells were seeded onto the six-well tissue culture plates $\left(1 \times 10^{5}\right.$ cells per well). The EdU (5-ethynyl-20-deoxyuridine) Apollo-488 In Vitro Imaging Kit (Ribo-Bio, Guangzhou, China) was utilized to quantify cell proliferation. Following treatment EdU $(2.5 \mu \mathrm{M})$ was added to RCC/epithelial cells for $6 \mathrm{~h}$. Cell nuclei were stained with Hoechst-33342 for $5 \mathrm{~min}$, visualized under a fluorescent microscope (Leica). We counted at least 400 cells of six random views to calculate EdU ratio for each treatment.

\section{In vitro cell migration and invasion assays}

As described ${ }^{16,17}$ RCC cells $\left(4 \times 10^{4}\right.$ cells of each condition in $200 \mu \mathrm{L}$ serum-free medium) were initially seeded onto the upper surfaces of "Transwell" chambers. The lower compartments were always filled with complete medium (containing 10\% FBS). Following $24 \mathrm{~h}$ incubation, the migrated cells on the lower surface were fixed, stained and counted. Matrigel (Sigma) was added in the chamber surfaces when analyzing cell invasion.

\section{Caspase activity assay}

Assaying of caspase-3/-9 activity was described previously $^{18}$. Twenty $\mu \mathrm{g}$ of cytosolic extracts of each treatment were added to the caspase assay buffer ${ }^{18}$ with the caspase- 3 substrate or the caspase- 9 substrate ${ }^{18}$. Release of 7-amido-4-(trifluoromethyl)-coumarin (AFC) was quantified by using a Fluoroskan system ${ }^{18}$. AFC optic density (OD) was recorded.

\section{Annexin V FACS assay}

As reported ${ }^{18}$, cells with the indicated treatment were washed and incubated with Annexin V-FITC $(10 \mu \mathrm{g} / \mathrm{mL})$ and propidium iodide (PI, $10 \mu \mathrm{g} / \mathrm{mL}$ ) (Invitrogen), and detected by fluorescence-activated cell sorting (FACS) using a Becton-Dickinson machine. Annexin V-positive cells were labeled as the apoptotic cells.

\section{TUNEL assay}

Cells were seeded onto the six-well tissue culture plates $\left(1 \times 10^{5}\right.$ cells per well). Following treatment, cells were incubated with TUNEL (Invitrogen, $10 \mu \mathrm{M}$ ) for $3 \mathrm{~h}$. Cell nuclei were stained with Hoechst-33342 for $5 \mathrm{~min}$, visualized under a fluorescent microscope (Leica). For each treatment, we counted at least 400 cells of six random views $(1 \times 100$ magnification $)$ to calculate TUNEL ratio.

\section{Western blotting assay}

Cells and tumor tissues were incubated with RIPA lysis buffer (Biyuntian). Thirty micrograms of lysates per lane 
were separated on $10 \%$ sodium dodecyl sulfate (SDS)polyacrylamide gel electrophoresis (PAGE) gels, and transferred to polyvinylidene difluoride (PVDF) membranes (Millipore). After blocking, the blots were incubated with the applied primary and secondary antibodies. The enhanced chemiluminescence (ECL) reagents (GE Healthcare) were added to the blots to detect the targeted protein bands. Quantification of the band intensity was performed with Quantity One 4.6.2 software (Bio-Rad, Hercules, CA).

\section{Reactive oxygen species (ROS) assay}

As described $^{19}$, the ROS levels were tested by using the carboxy-H2DCFDA dye. Following treatment, cells were stained with carboxy-H2-DCFDA $(10 \mu \mathrm{M})$ for $30 \mathrm{~min}$ under the dark. The DCF fluorescence was measured under $485 \mathrm{~nm}$ excitation and $525 \mathrm{~nm}$ emission using the Fluorescence machine (Thermo Scientific, Shanghai, China).

\section{Glutathione content assay}

Reduced glutathione (GSH) is one key scavenger of ROS, and its ratio with oxidized disulfide form glutathione (GSSG) can be used as a quantitative indicator of oxidative stress intensity ${ }^{20}$. Following treatment, cells were lysed. The ratio of reduced to oxidized glutathione (GSH/ GSSG) was measured using the GSH/GSSG assay kit (Beyotime).

\section{Sphingosine kinase 1 (SphK1) activity assay}

For each treatment, $200 \mu \mathrm{g}$ lysates were incubated with $25 \mu \mathrm{M}$ D-erythro-sphingosine in $0.1 \%$ Triton X-100, $2 \mathrm{mM}$ ATP, and $\left[{ }^{\gamma-32} \mathrm{P}\right]$ ATP for $30 \mathrm{~min}$ at $37^{\circ} \mathrm{C}^{21}$, stopped by adding $20 \mu \mathrm{L}$ of $\mathrm{HCl}$, plus $800 \mu \mathrm{L}$ of chloroform/methanol/ $\mathrm{HCl}$ (100:200:1, v/v). Afterwards, chloroform and $\mathrm{KCl}$ ( $250 \mu \mathrm{L}$ each) were added, and centrifugation was performed to separate layer phases. The organic layer was dried and resuspended in chloroform $/$ methanol/ $/ \mathrm{HCl}^{21}$. Lipids were resolved on silica TLC plates in 1-butanol/acetic acid/ water $^{21}$. Labeled sphingosine-1-phosphate (S1P) spots were visualized by autoradiography and quantified by scraping and counting in a scintillation counter. SphK1 activity was evaluated as $\mathrm{pmol} / \mathrm{h} / \mathrm{g}$ protein.

\section{Ceramide content assay}

The cellular ceramide level was analyzed by the protocol reported early ${ }^{22}$, tested as fmol by nmol of phospholipids.

\section{Mitochondrial depolarization}

As described ${ }^{23}$ following stress-induced mitochondrial depolarization, JC-1 dye shall aggregate in mitochondria, forming green monomers ${ }^{24}$. RCC were seeded onto the 24-well tissue-culturing plates $\left(1 \times 10^{4}\right.$ cells per well). Following SC66 treatment cells were incubated with JC-1
$(5 \mu \mathrm{g} / \mathrm{mL})$ for $30 \mathrm{~min}$, washed and tested immediately under a fluorescence spectrofluorometer at $550 \mathrm{~nm}$.

\section{AKT1 short hairpin RNA (shRNA)}

AKT1 shRNA lentivirus (sc-29195V, Santa Cruz Biotech, $10 \mu \mathrm{L} / \mathrm{mL}$ medium) was added to $786-\mathrm{O}$ cells for $24 \mathrm{~h}$. Stable cells were selected by puromycin $(5.0 \mu \mathrm{g} / \mathrm{mL})$ for another 10 days. Expression of AKT1 in the stable cells was determined by Western blotting assay.

\section{AKT knockout}

The small guide RNA (sgRNA) targeting human AKT1 (Target DNA sequence, 5'-TCACGTTGGTCCACATCCTG) was inserted into the lenti-CRISPR-GFPpuro plasmid ${ }^{25}$. The construct was then transfected to 786-O cells by Lipofectamine 2000. FACS was performed to sort the GFP-positive 786-O cells. The resulting single cells were further cultured in the selection medium with puromycin $(5 \mu \mathrm{g} / \mathrm{mL})$ for 10 days. AKT1 knockout in stable cells was verified by Western blotting assay.

\section{Xenograft model}

Female CB-17 severe combined immunodeficiency disease (SCID) mice, $4-5$ week old, $17-18 \mathrm{~g}$, were provided by the Animal Center of Soochow University (Suzhou, China). $786-O$ cells $\left(6 \times 10^{6}\right.$ per mouse, in $200 \mu \mathrm{L}$ DMEM/ Matrigel, no serum) were subcutaneously (s.c.) injected into flanks. After three week, the xenografts, close to $100 \mathrm{~mm}^{3}$, were established ("Day-0"). Ten mice per group were treated once daily by gavage with either vehicle control or SC66 (10 or $25 \mathrm{mg} / \mathrm{kg}$ body weight) for 24 consecutive days. Every six days, the mice body weights and bi-dimensional tumor measurements ${ }^{18}$ were recorded. The animal protocol was approved by the Institutional Animal Care and Use Committee (IACUC) of Soochow University and Ethics Review Board of Soochow University (Suzhou, China).

\section{Statistical analysis}

The investigators were blinded to the group allocation during all experiments. Results were expressed as the mean \pm standard deviation (SD). Statistical analysis among different groups was performed via one-way analysis of variance (ANOVA) with Scheffe's test using SPSS20.0 software (SPSS Inc., Chicago, IL). The two-tailed unpaired $T$ test (Excel 2007) was applied to test the significance of the difference between two treatment groups. $P$ values of $<0.05$ were considered statistically significant.

\section{Results \\ SC66 inhibits RCC cell progression in vitro}

To study the mechanism of SC66 cytotoxicity cultured human RCC786-O cells ${ }^{8-10}$ were treated with different concentrations of SC66. The MTT assay of cell viability 
demonstrated that SC66 dose-dependently reduced the viability of 786-O cells (Fig. 1a), in a time-dependent manner that required at least $48 \mathrm{~h}$ to exert a significant effect (Fig. 1a). The IC-50 of SC66 was close to $3 \mu \mathrm{M}$ at $72 \mathrm{~h}$ and $96 \mathrm{~h}$ (Fig. 1a), and soft agar colony studies demonstrated that SC66 $(1-30 \mu \mathrm{M})$ significantly decreased the number of viable786-O cell colonies (Fig. 1b). Examining 786-O cell proliferation, both BrdU ELISA and EdU staining confirmed that SC66 inhibited nuclear BrdU incorporation (Fig. 1c) and EdU incorporation (Fig. 1d) in a dose dependent manner. Measuring cell migration and invasion, Transwell and Matrigel Transwell assays, respectively, demonstrated that SC66 $(3 \mu \mathrm{M}, 24 \mathrm{~h})$ potently inhibited 786-O cell migration (Fig. 1e) and invasion (Fig. 1f) in vitro. Similar results were obtained with the A498 human RCC cell line ${ }^{8,9}$, where SC66 $(3 \mu \mathrm{M}$, $48 / 72 \mathrm{~h}$ ) decreased cell viability (Fig. S1A) and proliferation (Fig. S1B), and inhibited A498 cell migration and invasion (Fig. S1C, D).

In the primary human RCC cells, derived from three RCC patients ("RCC1/RCC2/RCC3"), SC66 potently reduced viability (Fig. 1g) and decreased proliferation (Fig. 1h). Transwell results, Fig. 1i, showed that SC66 (3 $\mu \mathrm{M}$, $24 \mathrm{~h}$ ) significantly decreased the number of migrated RCC cells. In contrast, immortalized HK-2 tubular epithelial cells $^{26,27}$ and the primary human renal epithelial cells ("Ren-Epi", from Dr. $\mathrm{Hu}^{28}$ ) were resistant to SC66, showing no significant effect on viability, proliferation or migration (Fig. 1j-l).

\section{SC66 provokes apoptosis activation in RCC cells}

Using the previously described methods ${ }^{8-10,15}$, we tested the effect of SC66 on cell apoptosis. As shown, SC66 dose-dependently increased the activities of caspase- 3 and caspase-9 in 786-O cells (Fig. 2a). Analyzing apoptosisassociated proteins, SC66 $(1-10 \mu \mathrm{M})$ induced cleavage of caspase-3, caspase-9, and PARP [poly (ADP-ribose) polymerase], and downregulatedBcl-2 (Fig. 2b). Annexin $\mathrm{V}$ FACS assay results demonstrated that $\mathrm{SC66}(3 \mu \mathrm{M})$ mainly induced apoptosis (Annexin $\mathrm{V}^{+/+}$) in 786-O cells (Fig. 2c). Furthermore, the percentage of cells with positive nuclear TUNEL staining was significantly increased following SC66 treatment (Fig. 2d). Significantly, cotreatment of the caspase-3 inhibitor z-DEVD-cho or the pan caspase inhibitor $\mathrm{z}$-VAD-cho largely attenuated the SC66 $(3 \mu \mathrm{M}, 72 \mathrm{~h})$-induced viability reduction in $786-\mathrm{O}$ cells (Fig. 2e). Similar results were observed in the A498 cell line (Fig. S1E-S1I). In primary human RCC cells ("RCC1/RCC2/RCC3"), treatment with SC66 induced apoptosis activation, as evidenced by a significant increase in nuclear TUNEL staining (Fig. 2f). In line with the above results showing that immortalized HK-2 tubular and primary renal epithelial cells are resistant to SC66, no significant apoptosis was detected (Fig. 2g).

\section{SC66 inhibits AKT-mTOR activation in RCC cells}

As SC66 is reported to inhibit Akt in hepatocellular carcinoma cells ${ }^{11,12,29}$, we tested AKT and mTOR signaling in SC66-treated RCC cells. Western blot results demonstrated that phosphorylation of AKT (at both Ser473 and Thr-308) and S6K1 (at Ser-389) were inhibited by SC66 $(3 \mu \mathrm{M}, 2 \mathrm{~h})$ in both $786-\mathrm{O}$ and primary RCC cells ("RCC1/RCC2") (Fig. 3a). These results confirm that that SC66 acts to block AKT, mTORC1 (indicated by p$\mathrm{S} 6 \mathrm{~K} 1^{30,31}$ ) and mTORC2 (indicated by $\mathrm{p}-\mathrm{AKT}$ at Ser $473^{30,31}$ ) in RCC cells (Fig. 3a). Total AKT1 protein level was also decreased by SC66 treatment in RCC cells (Fig. $3 a)$, possibly due to ubiquitin-mediated degradation ${ }^{11}$. Quantified results, integrating five sets of repeated blotting data in 786-O and primary RCC cells, show that SC66-induced significant AKT-mTORC1/2 inhibition (Fig. 3c-e). Basal AKT-mTORC1/2 activity was significantly lower in the primary renal epithelial cells (Fig. $3 \mathrm{~b}-\mathrm{e})$, possibly explaining the ineffectiveness of this compound on normal epithelial cells (Figs. 1 and 2).

As Erk signaling plays a role in RCC oncogenesis ${ }^{13,32-34}$, we examinedp-Erk1/2 at Thr202/Tyr204, finding that it was unchanged followingSC66 $(3 \mu \mathrm{M}, 2 \mathrm{~h})$ treatment in 786-O and primary RCC cells (Fig. 3f). However, cotreatment with the Erk inhibitors, PD98059 and U0126, significantly potentiated SC66-induced viability reduction (Fig. 3g) and apoptosis (Fig. 3h). Treatment with the Erk1/ 2 inhibitors alone induced minor but significant cytotoxicity in the tested RCC cells (Fig. 3g, h). These results suggest that Erk inhibition could sensitize SC66-induced cytotoxicity in human RCC cells.

\section{SC66 induces oxidative stress, SphK1 inhibition, and JNK activation in RCC cells}

To examine whether SC66-induced cytotoxicity is primarily via Akt inhibition, we compared SC66 efficacy with known AKT inhibitors, including the AKT specific inhibitor MK$2206^{35}$ and the PI3K-AKT-mTOR pan inhibitor LY294002 ${ }^{36}$. MTT assay results (Fig. 4a) demonstrate that SC66 was more potent than MK-2206 and LY294002 in inhibiting786-O cell viability. Significantly, SC66 further reduced the viability of 786-O cells pretreated with MK-2206 and LY294002 (Fig. 4a), suggesting that SC66 effects are not limited to AKT inhibition. To confirm an AKT-independent mechanism of SC66 cytotoxicity in 786-O cells, shRNA and CRISPR/Cas9 methods were applied to silence and knockout AKT1, respectively (Fig. 4b, the upper panel). Results show that SC66 was cytotoxic in AKT1-silenced/-KO cells (Fig. 4b), indicating AKTindependent mechanisms of killing 786-O cells. 


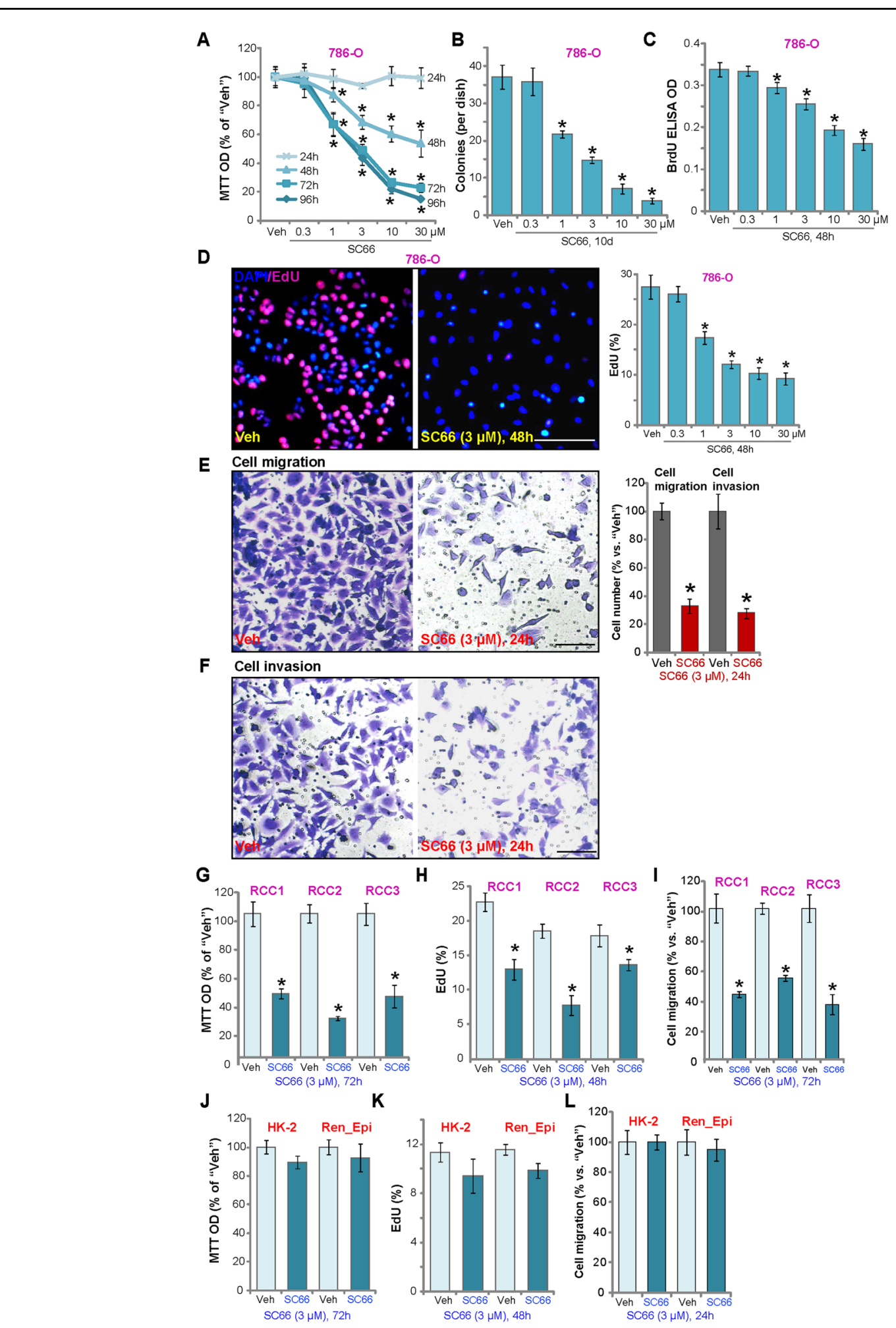

Fig. 1 SC66 inhibits RCC cell progression in vitro. 786-O RCC cells (a-f), primary human RCC cells ("RCC1/RCC2/RCC3", g-i), or HK-2 tubular epithelial cells $\mathbf{j} \mathbf{j} \mathbf{I})$, the primary human renal epithelial cells ("Ren_Epi") $(\mathbf{j}-\mathbf{I})$ were treated with indicated concentration of SC66, cells were further cultured for applied time periods, cell functions, including cell survival, proliferation, migration and invasion were tested by the appropriate assays. For each assay, $n=5$. Data were expressed as the mean \pm standard deviation (S.D.). ${ }^{*} P<0.05$ vs. DMSO (0.1\%) vehicle ("Veh", same for all Figures). In this figure, experiments were repeated three times, and similar results were obtained each time. Bar $=100 \mu m(\mathbf{d}-\mathbf{f}, \mathbf{h})$. 


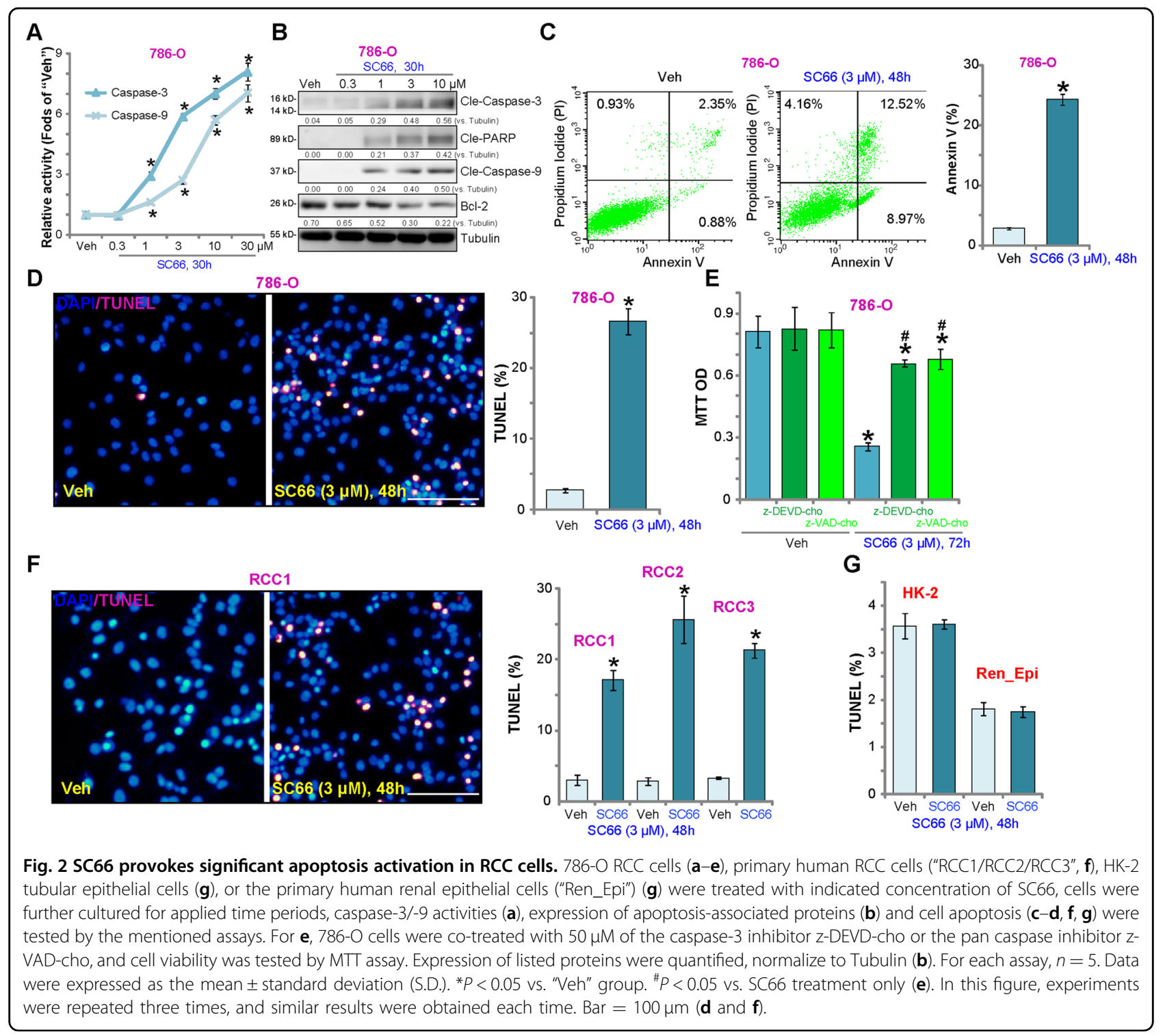

Exploring possible AKT-independent mechanisms, we found that SC66 induced oxidative stress in 786-O cells, evidenced by increased ROS production (DCF-DA intensity increase, Fig. 4c) and reduced GSH/GSSG ratio (Fig. 4d). In addition, SC66 increased mitochondrial depolarization, tested by JC-1 green monomers formation (Fig. 4e). Furthermore, sphingosine kinase 1 (SphK1) activity, an enzyme that prevents ceramide accumulation, was inhibited in SC66-treated 786-O cells (Fig. 4f) with a concomitant increase in ceramide levels (Fig. 4g). A consequence of ceramide accumulation is pro-apoptotic JNK activation ${ }^{37}$, and a significant increase of JNK1/2 phosphorylation was detected in SC66-treated cells, confirming JNK activation (Fig. 4h). To examine whether these pathways are involved in the cytotoxic action of SC66, we tested the effects of the ROS scavenger NAC, the JNK inhibitor JNKi, and anti-ceramide sphingolipidsphingosine-1-phosphate (S1P). As shown, SC66-induced viability reduction (Fig. 4i) and apoptosis (Fig. 4j) were inhibited by pretreatment with NAC, JNKi or S1P. In the primary human RCC cells ("RCC1"), SC66 treatment similarly reduced ROS production (Fig. 4k), mitochondrial depolarization (Fig. 4l), ceramide accumulation(Fig. 4m) and JNK activation (Fig. 4n). Thus, AKT-independent mechanisms participated in SC66induced cytotoxicity in RCC cells.

\section{SC66 inhibits 786-O xenograft tumor growth in SCID mice}

We tested the potential effect of SC66 in vivo using the previously-described $786-\mathrm{O}$ xenograft tumor model ${ }^{8,9}$. 786-O cells were s.c. injected into the flanks of SCID mice and xenografts established within three weeks when 

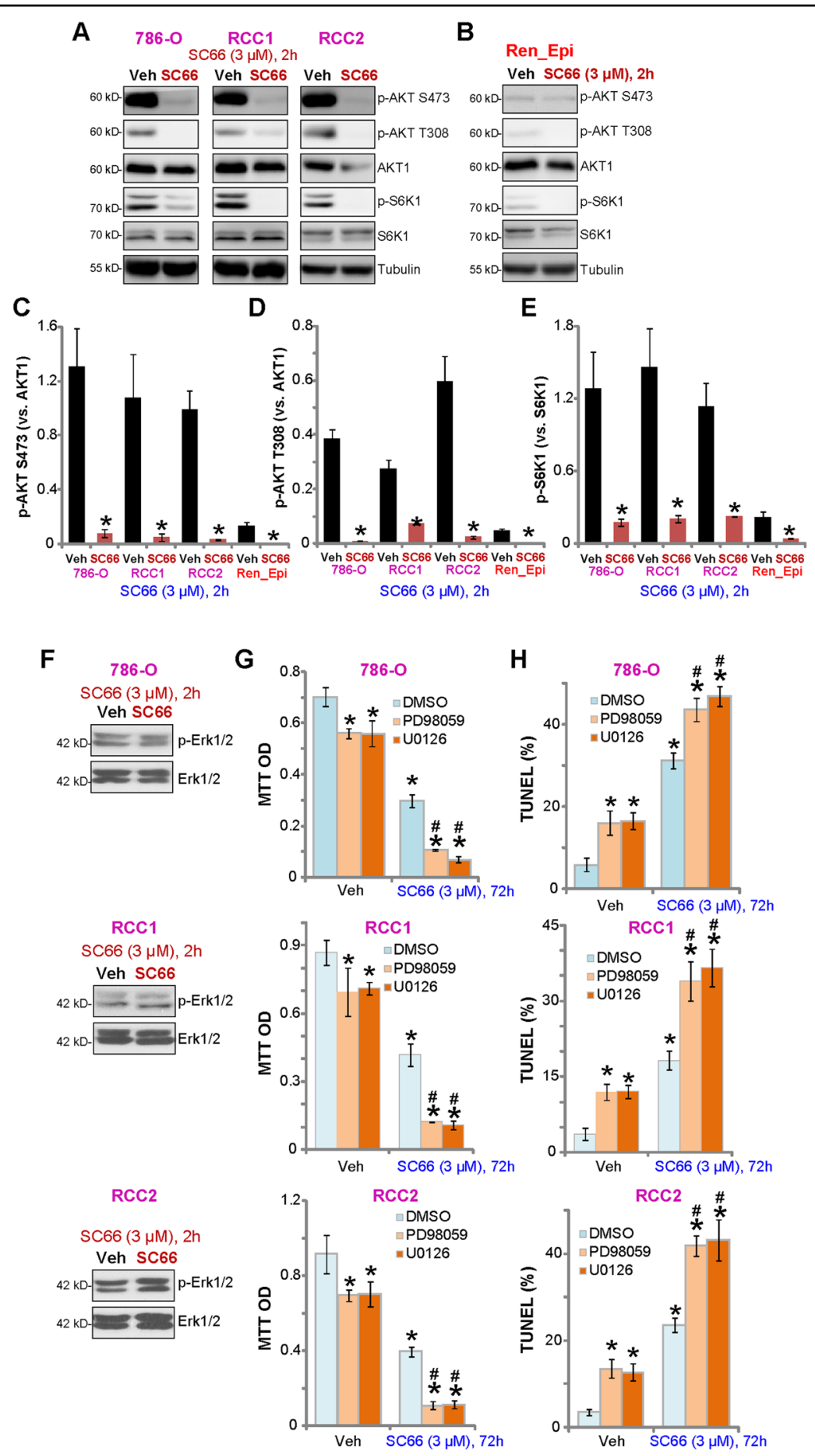

Fig. 3 SC66 inhibits AKT-mTOR activation in RCC cells. 786-O cells, the primary human RCC cells ("RCC1/2"), or the renal epithelial cells ("Ren_Epi") were treated with SC66 $(3 \mu \mathrm{M})$ for $2 \mathrm{~h}$, expression of listed proteins in total cell lysates were tested by Western blotting assay $(\mathbf{a}, \mathbf{b}$, and $\mathbf{f}$ ).The quantified results integrating five sets of blotting data were presented $(\mathbf{c}-\mathbf{e})$. $786-\mathrm{O}$ cells or RCC1/2 primary cells were treated with SC66 $(3 \mu \mathrm{M})$, together with or without Erk inhibitor PD98059/U0126 (each at $5 \mu \mathrm{M}$ ), cells were further cultured for $72 \mathrm{~h}$, and cell viability and apoptosis tested by MTT (g) and nuclear TUNEL staining (h) assays, respectively. Data were expressed as the mean \pm standard deviation (S.D.). "DMSO" stands for 0.1\% DMSO (g, h). * $P<0.05$ vs. "Veh" group. ${ }^{\#} P<0.05$ vs. "DMSO" plus SC66 treatment $(\mathbf{g}, \mathbf{h})$. In this figure, experiments were repeated three times, and similar results were obtained each time. 

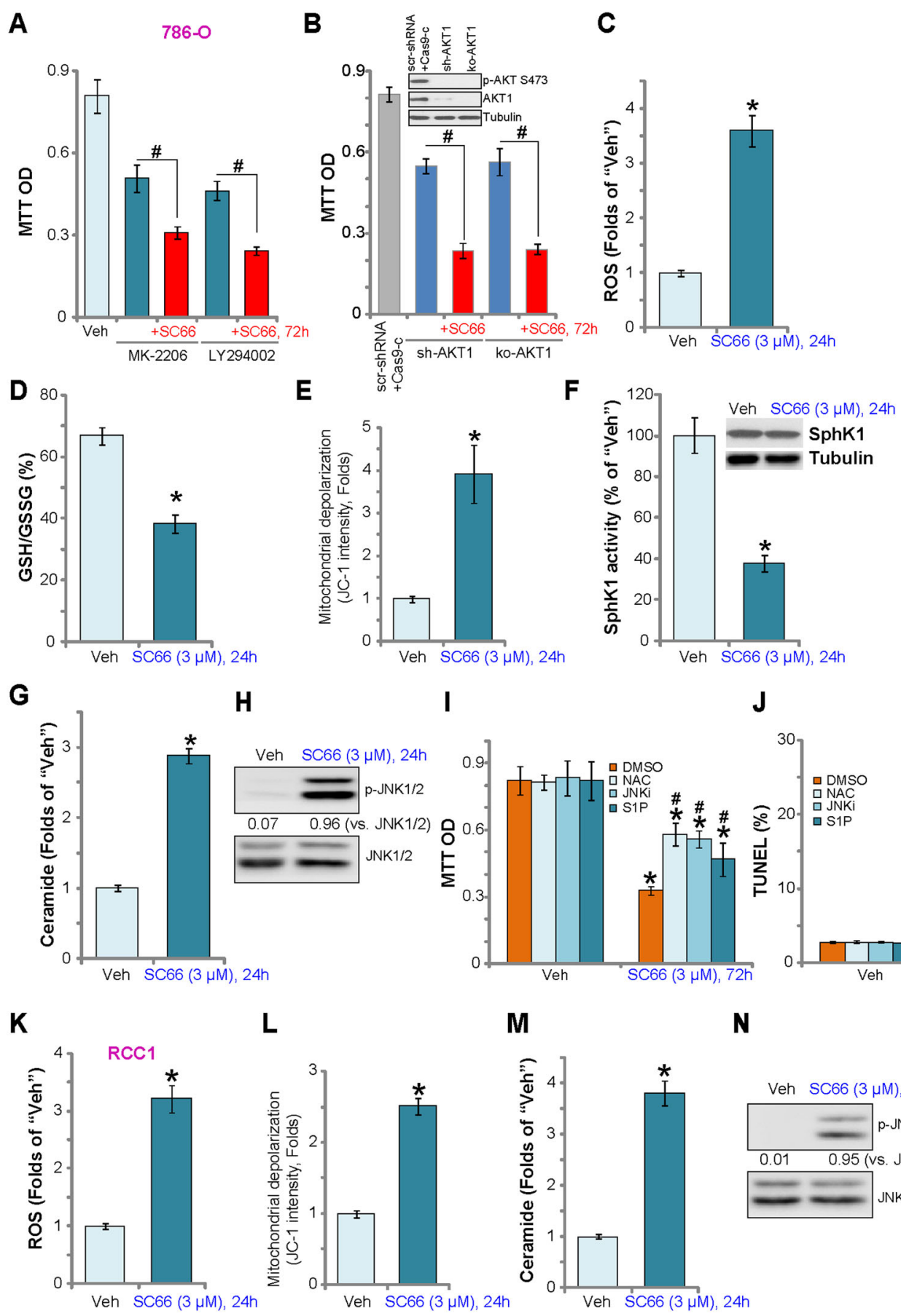

$\mathbf{L}$

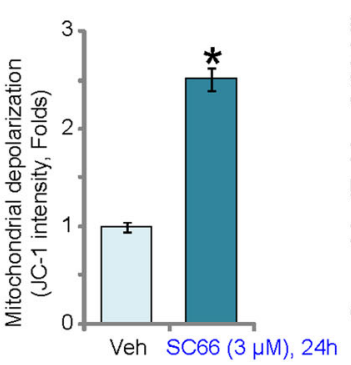

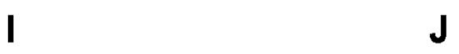
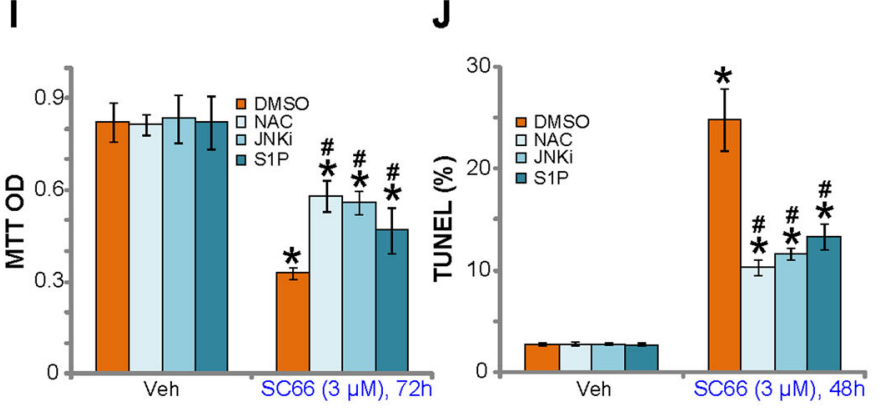

M

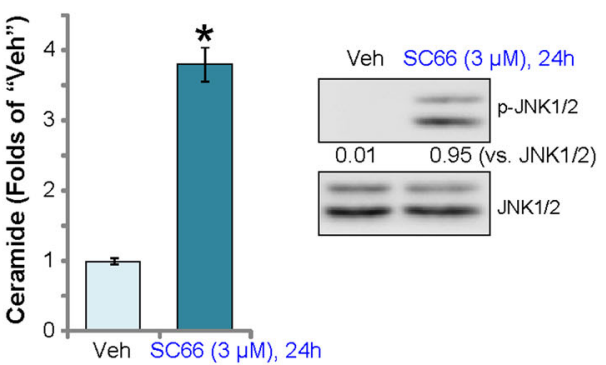

Fig. 4 SC66 induces oxidative stress, SphK1 inhibition, and JNK activation in RCC cells. 786-O cells were treated with MK-2206, LY294002 or plus SC66 (all at $3 \mu \mathrm{M}$ ), cells were further cultured, and cell viability was tested by MTT assay (a, 72 h). Stable 786-O cells with AKT1 shRNA ("sh-AKT1") or CRISPR/Cas9 AKT1-KO construct ("ko-AKT1"), as well as the control cells with scramble control shRNA and Cas9 empty plasmid ("scr-shRNA+ Cas9-c"), were tested by Western blotting assay of AKT expression (b, the upper panel), cells were treated with/without SC66 ( $3 \mu \mathrm{M})$ for $72 \mathrm{~h}$, cell

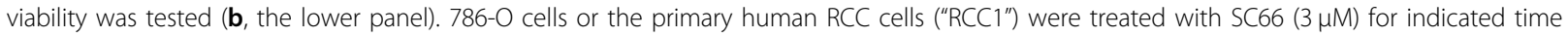
periods, ROS production (c and $\mathbf{k}$ ), GSH/GSSG ratio (d), mitochondrial depolarization (e and $\mathbf{I}$ ), SphK1 expression and activity (f), as well as the ceramide contents $(\mathbf{g}$ and $\mathbf{m})$ and p-/t-JNK expression (h and $\mathbf{n})$ were tested by the appropriate assays. 786-O cells were pretreated for 30 min with NAC $(400 \mu \mathrm{M})$, JNKi $(10 \mu \mathrm{M})$, or S1P $(10 \mu \mathrm{M})$, followed by SC66 $(3 \mu \mathrm{M})$ treatment for 48 and $72 \mathrm{~h}$, cell viability and apoptosis were tested by MTT assay (h) and TUNEL staining assay (i), respectively. Phosphorylated JNK1/2 was normalized to total JNK1/2 (h and $\mathbf{n}$ ). For each assay, $n=5$. Data were expressed as the mean \pm standard deviation (S.D.). ${ }^{*} P<0.05$ vs. "Veh" group. ${ }^{\#} P<0.05$ vs SC66 treatment only (i, $\mathbf{j}$ ). In this figure, experiments were repeated three times, and similar results were obtained each time. 

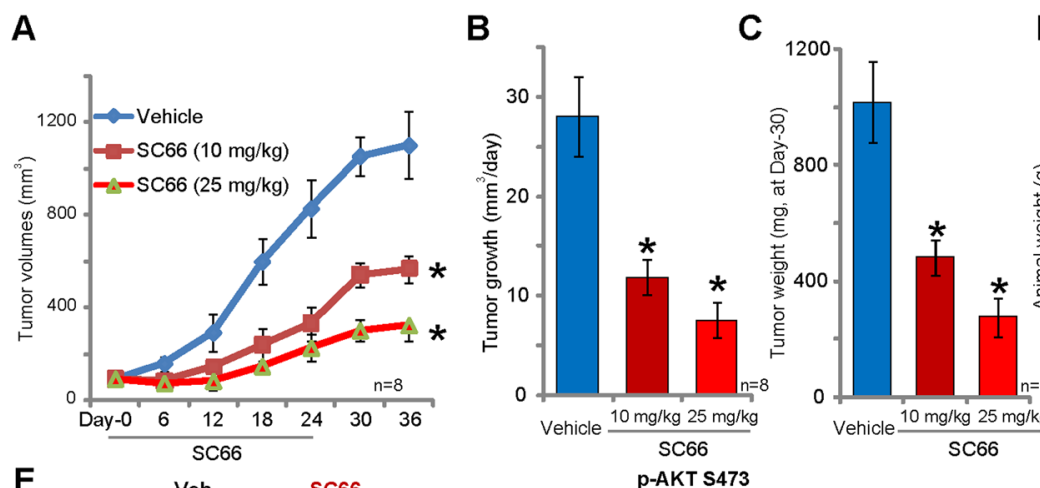

D

E
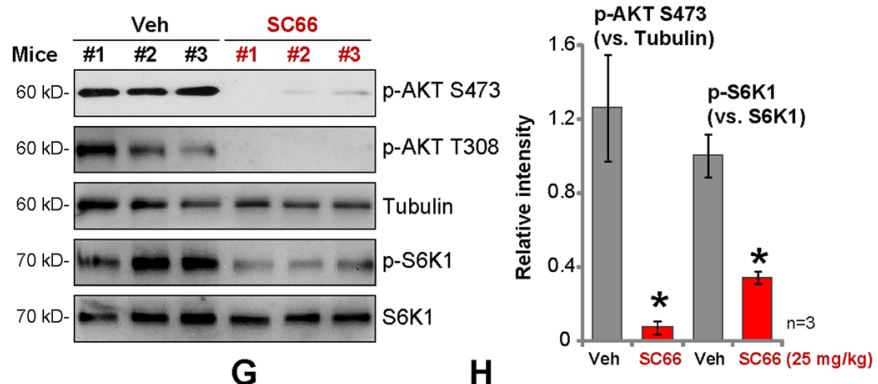

$\mathrm{F}$

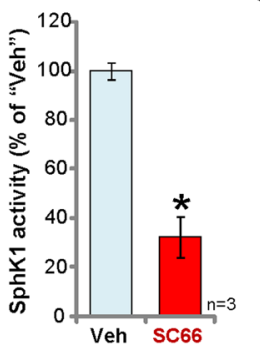

G

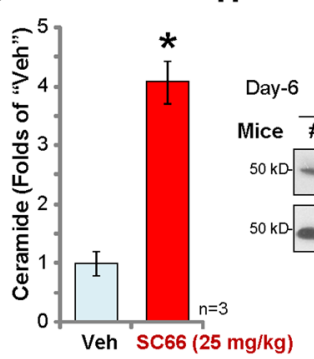

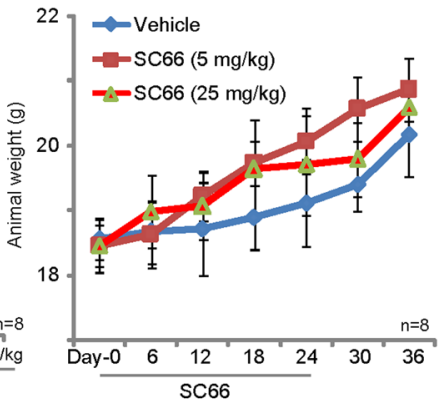

Fig. 5 SC66 inhibits 786-0 xenograft tumor growth in SCID mice. 786-O tumor-bearing SCID mice (eight mice per group, $n=8$ ) were orally administrated with SC66 (10/25 mg/kg body weight, daily, for 24 days) or vehicle control ("Vehicle"), tumor volumes (a) and mice body weights (d) were recorded every 6 days. Estimated daily tumor growth was calculated as described (b). At Day-36, tumors were isolated and weighted (c). At treatment Day-6, two hours after SC66 (25 mg/kg) or vehicle administration, three 786-0 tumors ( $n=3)$ of each group (total six tumors) were isolated; Expression of listed proteins in tumor lysates was tested by Western blotting assays (e and $\mathbf{h}$ ); The relative SphK1 activity (f) and ceramide contents $(\mathbf{g})$ in tumor lysates were tested as well. The quantified results integrating all three sets of blotting data were presented (e and $\mathbf{h}$ ). Data were expressed as the mean \pm standard deviation (S.D.). ${ }^{*} P<0.05$ vs. "Vehicle" group.

tumors were around $100 \mathrm{~mm}^{3}$ ("Day-0"). We found that oral administration of SC66, at 10 and $25 \mathrm{mg} / \mathrm{kg}$ body weight, significantly inhibited tumor volume(Fig. 5a), and that daily tumor growth was significantly inhibited (Fig. 5b). At Day-36, tumors from all three groups were isolated and weighted individually.SC66-treated 786-O tumors weighted significantly less than the vehicle control tumors (Fig. 5c), while mouse body weights were not significantly different between the three groups (Fig. 5d). At treatment Day-6, two hours after SC66 $(25 \mathrm{mg} / \mathrm{kg})$ or vehicle administration, three 786-O tumors from each group (total six tumors) were isolated. Analyzing signaling changes, AKT-S6K phosphorylation was significantly inhibited in SC66-treated tumor lysates (Fig. 5e), confirming AKT-mTOR inhibition. In line with the in vitro findings, SC66 treatment decreased SphK1 activity (Fig. 5f), increased ceramide levels (Fig. 5g), and increased JNK activation (Fig. 5h).

\section{Discussion}

Our study shows that SC66 inhibited cell viability, proliferation, migration and invasion in established (786$\mathrm{O}$ and A498 lines) and primary human RCC cells.SC66 was found to inhibit AKT-mTORC1/2 activation and induce significant apoptosis in RCC cells. In contrast, this AKT inhibitor was non-cytotoxic to HK-2 epithelial cells and primary human renal epithelial cells with low basal AKT-mTORC1/2 activation. In vivo, SC66 oral administration, at well-tolerated doses, potently inhibited subcutaneous 786-O xenograft growth in SCID mice. 
mTORC1 inhibitors are approved by FDA for the treatment of advanced RCC patients after failure of either sunitinib or sorafenib ${ }^{2,3}$. However, the use of mTORC1 inhibitors can have several drawbacks. First, the mTORC1 inhibitors, rapamycin and its analogs ("rapalogs"), only indirectly inhibit mTORC1 $1^{38,39}$. Second, mTORC1 inhibition can induce feedback activation of the PI3K-AKT and ERK-MAPK oncogenic pathways ${ }^{38-40}$. Third, rapalogs are unable to directly inhibit mTORC2, the latter being equally as important as mTORC1 in RCC progression. We have previously shown that WYE-687, a mTORC1/2 dual inhibitor, inhibited RCC cell growth with greater efficiency than mTORC1 inhibitors ${ }^{9}$. Further, the mTORC1/2 inhibitor, AZD2014, exerted more potent anti-RCC cell activity than rapalogs ${ }^{18}$. Similarly, the finding that SC66 can block AKT and mTORC1/2 activation in established and primary RCC cells is an advantage of this compound.

Furthermore, SC66 also exhibits cytotoxic actions independent of AKT1. Here, we show that in RCC cells SC66 induced ROS production, SphK1 inhibition, ceramide accumulation and JNK activation, which does not occur in RCC cells treated with the AKT specific inhibitor MK-2206 $6^{35,41}$ or in AKT1-silenced/-KO RCC cells. Significantly, the ROS scavenger NAC, the JNK inhibitor and anti-ceramide sphingolipid S1P all mitigated, but did not reverse, SC66-induced cytotoxicity in RCC cells. Importantly, confirming in vitro results, SphK1 inhibition, ceramide accumulation and JNK activation were detected in SC66-treated 786-O xenograft tumors. Therefore, SC66 acts through both AKT-dependent and AKT-independent mechanisms to exert more potent anti-RCC activity.

SphK1 is over-expressed and/or hyper-activated in RCC, promoting cancer progression ${ }^{42,43}$. SphK1 phosphorylates sphingosine to form $\mathrm{S}_{1}{ }^{44,45}$, and SphK1 inhibition or silencing induces ceramide accumulation to promote cell apoptosis. Despite the importance of sphingolipid-derived signaling in tumorigenesis, there is a lack of potent and selective inhibitors of SphK. We found that SC66 inhibits SphK1 activation leading to proapoptotic ceramide accumulation and JNK activation in vitro and in vivo. Further studies are needed to determine the mechanism by which SC66 inhibits SphK1 in RCC cells.

It has been shown that Erk activation contributes to everolimus-acquired resistance and a poor prognosis in RCC patients ${ }^{33}$. Contrarily, Erk inhibition enhanced the efficacy of everolimus against RCC cells ${ }^{33}$. Yuen et al., found that AZD6244, an Erk inhibitor, at low doses augmented the antitumor activity of sorafenib ${ }^{34}$. In this study, we show that inhibition of Erk by PD98059 or U0126 potentiated SC66-induced cytotoxicity and apoptosis in 786-O and primary RCC cells, indicating that Erk activation could be a key resistance mechanism of SC66 in RCC cells.

\section{Conclusion}

In summary, we show that SC66 inhibits RCC cell progression in vitro and in vivo, through AKT-dependent and AKT-independent mechanisms. It should be noted that the findings of in vitro and animal RCC studies could not be directly translated to humans, and thus the efficacy and safety of SC66 need to be further investigated and confirmed.

\section{Acknowledgements \\ This study was supported by the National Natural Science Foundation of China (81472776, 81773221, and 81773192), the Natural Science Foundation of Jiangsu Province (BK20161222, BK20171248), by Suzhou Science and Technology Planed Projects (SYS201629), and the grant for Key Young Talents of Medicine in Jiangsu (QNRC2016875 and QNRC2016527), by Foundation of tumor clinical and basic research team of Affiliated Kunshan Hospital of Jiangsu University (KYC005) and by Jiangsu Province"333 Project" Research Projects (2016-III-0367).}

\section{Author details}

'Department of Urology, The Second Affiliated Hospital of Soochow University, Suzhou, China. ${ }^{2}$ Institute of Neuroscience, Soochow University, Suzhou, China. ${ }^{3}$ Department of Radiotherapy and Oncology, Affiliated Kunshan Hospital of Jiangsu University, Suzhou, China. ${ }^{4}$ Department of Urology, Ningbo Urology Nephrology Hospital, Ningbo, China

Conflict of interest

The authors declare that they have no conflict of interest.

\section{Publisher's note}

Springer Nature remains neutral with regard to jurisdictional claims in published maps and institutional affiliations.

Supplementary Information accompanies this paper at (https://doi.org/ 10.1038/s41419-020-2566-1).

Received: 14 February 2020 Revised: 17 April 2020 Accepted: 20 April 2020 Published online: 11 May 2020

\section{References}

1. Siegel, R. L., Miller, K. D. \& Jemal, A. Cancer statistics, 2018. CA Cancer J. Clin. 68, 7-30 (2018).

2. Pal, S. K. \& Quinn, D. I. Differentiating mTOR inhibitors in renal cell carcinoma. Cancer Treat. Rev. 39, 709-719 (2013).

3. Husseinzadeh, H. D. \& Garcia, J. A. Therapeutic rationale for mTOR inhibition in advanced renal cell carcinoma. Curr. Clin. Pharmacol. 6, 214-221 (2011).

4. Figlin, R. A., Kaufmann, I. \& Brechbiel, J. Targeting PI3K and mTORC2 in metastatic renal cell carcinoma: new strategies for overcoming resistance to VEGFR and mTORC1 inhibitors. Int. J. Cancer 133, 788-796 (2013).

5. Burgio, S. L. et al. Perspectives on mTOR inhibitors for castration-refractory prostate cancer. Curr. Cancer Drug Targets 12, 940-949 (2012).

6. Konings, I. R., Verweij, J., Wiemer, E. A. \& Sleijfer, S. The applicability of mTOR inhibition in solid tumors. Curr. Cancer Drug Targets 9, 439-450 (2009).

7. Motzer, R. J. et al. Efficacy of everolimus in advanced renal cell carcinoma: a double-blind, randomised, placebo-controlled phase III trial. Lancet $\mathbf{3 7 2}$, 449-456 (2008).

8. Zhu, H. et al. Dual inhibition of BRD4 and PI3K-AKT by SF2523 suppresses human renal cell carcinoma cell growth. Oncotarget 8, 98471-98481 (2017).

9. Pan, X. D. et al. Concurrent inhibition of mTORC1 and mTORC2 by WYE-687 inhibits renal cell carcinoma cell growth in vitro and in vivo. PLOS ONE 12, e0172555 (2017).

10. Gu, D. H. et al. microRNA-302c-3p inhibits renal cell carcinoma cell proliferation by targeting Grb2-associated binding 2 (Gab2). Oncotarget 8, 26334-26343 (2017). 
11. Jo, $\mathrm{H}$. et al. Deactivation of Akt by a small molecule inhibitor targeting pleckstrin homology domain and facilitating Akt ubiquitination. Proc. Natl Acad. Sci. USA 108, 6486-6491 (2011)

12. Cusimano, A. et al. Cytotoxic activity of the novel small molecule AKT inhibitor SC66 in hepatocellular carcinoma cells. Oncotarget 6, 1707-1722 (2015).

13. Chen, C. M. et al. Alpha-Mangostin suppresses the metastasis of human renal carcinoma cells by targeting MEK/ERK expression and MMP-9 transcription activity. Cell Physiol. Biochem. 44, 1460-1470 (2017).

14. Wu, X. et al. Inhibition of BRD4 suppresses cell proliferation and induces apoptosis in renal cell carcinoma. Cell Physiol. Biochem. 41, 1947-1956 (2017)

15. Zheng, B. et al. MiRNA-30a-mediated autophagy inhibition sensitizes renal cell carcinoma cells to sorafenib. Biochem. Biophys. Res. Commun. 459, 234-239 (2015).

16. Wang, S. S. et al. Triptonide inhibits human nasopharyngeal carcinoma cell growth via disrupting Lnc-RNA THOR-IGF2BP1 signaling. Cancer Lett. 443 13-24 (2019).

17. LV, Y. et al. TBX2 over-expression promotes nasopharyngeal cancer cell proliferation and invasion. Oncotarget 8, 52699-52707 (2017).

18. Zheng, B. et al. Pre-clinical evaluation of AZD-2014, a novel mTORC1/2 dual inhibitor, against renal cell carcinoma. Cancer Lett. 357, 468-475 (2015).

19. Di, G., Wang, Z., Wang, W., Cheng, F. \& Liu, H. AntagomiR-613 protects neuronal cells from oxygen glucose deprivation/re-oxygenation via increasing SphK2 expression. Biochem. Biophys. Res. Commun. 493, 188-194 (2017).

20. Zitka, O. et al. Redox status expressed as GSH:GSSG ratio as a marker for oxidative stress in paediatric tumour patients. Oncol. Lett. 4, 1247-1253 (2012).

21. Lu, P. H. et al. Identification of sphingosine kinase 1 (SphK1) as a primary target of icaritin in hepatocellular carcinoma cells. Oncotarget 8, 22800-22810 (2017).

22. Gong, L. et al. Bortezomib-induced apoptosis in cultured pancreatic cancer cells is associated with ceramide production. Cancer Chemother. Pharmacol. 73, 69-77 (2014).

23. Hong, $\mathrm{H}$. et al. Dysregulation of CPWWP2A-miR-579 axis mediates dexamethasone-induced cytotoxicity in human osteoblasts. Biochem Biophys. Res. Commun. 517, 491-498 (2019).

24. Brooks, M. M., Neelam, S., Fudala, R., Gryczynski, I. \& Cammarata, P. R. Lenticular mitoprotection. Part A: monitoring mitochondrial depolarization with JC-1 and artifactual fluorescence by the glycogen synthase kinase-3beta inhibitor, SB216763. Mol. Vis. 19, 1406-1412 (2013).

25. Wu, F. et al. miR-1273g silences MAGEA3/6 to inhibit human colorectal cancer cell growth via activation of AMPK signaling. Cancer Lett. 435, 1-9 (2018).

26. Ryan, M. J. et al. HK-2: an immortalized proximal tubule epithelial cell line from normal adult human kidney. Kidney Int. 45, 48-57 (1994).

27. Komoike, Y., Inamura, H. \& Matsuoka, M. Effects of salubrinal on cadmiuminduced apoptosis in HK-2 human renal proximal tubular cells. Arch. Toxicol. 86, 37-44 (2012).

28. Ye, X. T., Huang, H., Huang, W. P. \& Hu, W. L. LncRNA THOR promotes human renal cell carcinoma cell growth. Biochem. Biophys. Res. Commun. 501, 661-667 (2018).
29. Tran, H. T. \& Zhang, S. Accurate prediction of the bound form of the Akt pleckstrin homology domain using normal mode analysis to explore structural flexibility. J. Chem. Inf. Model. 51, 2352-2360 (2011).

30. Vivanco, I. \& Sawyers, C. L. The phosphatidylinositol 3-Kinase AKT pathway in human cancer. Nat. Rev. Cancer 2, 489-501 (2002).

31. Sabatini, D. M. mTOR and cancer: insights into a complex relationship. Nat. Rev. Cancer 6, 729-734 (2006).

32. Bitzer, M. et al. Resminostat in combination with sorafenib as second-line therapy of advanced hepatocellular carcinoma-the SHELTER study. J Hepatol. 65, 280-288 (2016).

33. Zou, Y. et al. ERK inhibitor enhances everolimus efficacy through the attenuation of dNTP pools in renal cell carcinoma. Mol. Ther. Nucleic Acids 14 550-561 (2019).

34. Yuen, J. S. et al. Combination of the ERK inhibitor AZD6244 and low-dose sorafenib in a xenograft model of human renal cell carcinoma. Int. J. Oncol. 41, 712-720 (2012)

35. Hirai, H. et al. MK-2206, an allosteric Akt inhibitor, enhances antitumor efficacy by standard chemotherapeutic agents or molecular targeted drugs in vitro and in vivo. Mol. Cancer Ther. 9, 1956-1967 (2010).

36. Brunn, G. J. et al. Direct inhibition of the signaling functions of the mammalian target of rapamycin by the phosphoinositide 3-kinase inhibitors, wortmannin and LY294002. EMBO J. 15, 5256-5267 (1996).

37. Verheij, M. et al. Requirement for ceramide-initiated SAPKJJNK signalling in stress-induced apoptosis. Nature 380, 75-79 (1996).

38. Saxton, R. A. \& Sabatini, D. M. mTOR signaling in growth, metabolism, and disease. Cell 168, 960-976 (2017).

39. Lamming, D. W., Ye, L., Sabatini, D. M. \& Baur, J. A. Rapalogs and mTOR inhibitors as anti-aging therapeutics. J. Clin. Invest. 123, 980-989 (2013).

40. Shi, Y., Yan, H., Frost, P., Gera, J. \& Lichtenstein, A. Mammalian target of rapamycin inhibitors activate the AKT kinase in multiple myeloma cells by up-regulating the insulin-like growth factor receptor/insulin receptor substrate-1/phosphatidylinositol 3-kinase cascade. Mol. Cancer Ther. 4, 1533-1540 (2005).

41. Yap, T. A. et al. First-in-man clinical trial of the oral pan-AKT inhibitor MK2206 in patients with advanced solid tumors. J. Clin. Oncol. 29, 4688-4695 (2011).

42. Pchejetski, D. et al. FTY720 (fingolimod) sensitizes prostate cancer cells to radiotherapy by inhibition of sphingosine kinase-1. Cancer Res. 70, 8651-8661 (2010).

43. Ader, I., Brizuela, L., Bouquerel, P., Malavaud, B. \& Cuvillier, O. Sphingosine kinase 1: a new modulator of hypoxia inducible factor 1alpha during hypoxia in human cancer cells. Cancer Res. 68, 8635-8642 (2008).

44. Vadas, M., Xia, P., McCaughan, G. \& Gamble, J. The role of sphingosine kinase 1 in cancer: oncogene or non-oncogene addiction? Biochim. Biophys. Acta 1781, 442-447 (2008).

45. Shida, D., Takabe, K., Kapitonov, D., Milstien, S. \& Spiegel, S. Targeting SphK1 as a new strategy against cancer. Curr. Drug Targets 9, 662-673 (2008). 